\title{
Limitation in the use of drain tracing dye in the diagnosis of water seepage
}

Received: 3rd January, 2006

\section{Malcolm Hollis}

is Professor of Building Pathology at The University of Reading as well as being a practitioner. Malcolm Hollis Chartered Surveyors is a surveying practice with representation throughout England.

\begin{abstract}
Drain dye is designed for use in identifying the linkage between manholes, gullies or other connections. However, it has been the practice for drain dye to be used by surveyors to trace leakage of drains. There has been no research into the suitability of drain dyes for this alternative use. This paper deals with a design of a test to determine the effect of dyed water passing through simulated ground. The result of this test was that the colorant was removed from the water when dyed water was passed through $600 \mathrm{~mm}$ of sand. Further tests using different ground conditions are to be made.
\end{abstract}

\section{Keywords:}

dampness, diagnostics, drain dyes

\section{BACKGROUND}

The cause of water seepage within a building can be difficult to diagnose especially if the part of the building into which the water is seeping is below ground. The options for the source of the water that is penetrating could include ground water, a leaking pipe, drain fault or a myriad of other possibilities. In the past it has been common for surveyors to use drain dyes to test the possibility of the water seeping having come from underground drainage. Dye is mixed with water and poured into the drains and the seepage into the damp part of the building is monitored for any signs of the colour as part of the dampness visible. This process relies upon one key factor: the colour in the dye used not being affected by the dyed water's passage through the ground.

The colours in the majority of drain dyes are made from 100 per cent food-grade products, except for the colour green which contains, in most cases, 50 per cent fluoresceine. Dyes are non-toxic and odourless. They are approved by the national rivers authorities, water companies

Malcolm Hollis Chartered Surveyors 6 Rydal Road

London SW16 1 QN, UK

Tel: +44 (o) 2087699927

Fax: + 44 (o) 2087692670

E-mail: mh@malcolmhollis.org and local authorities. The dye is usually mixed in a bucket of cold water which is then added to the flow of water in the drain or pipe being checked.

The verification of flow from a tested source is done by recognising the colour in the water flowing into the area being tested as being the same 
as that used in the dyed water poured into the pipework suspected of being the source of the leaking water.

\section{TESTING THE EFFECT OF DRAIN DYE AND ITS PASSAGE THROUGH THE GROUND}

\section{Methodology}

To test the effect of ground conditions upon dyed water a $65 \mathrm{~mm}-\mathrm{long}$, $110 \mathrm{~mm}$-diameter, PVCu solid-walled pipe was selected and fixed in a vertical position (Figure 1). This was filled with white-washed soft sand to within $50 \mathrm{~mm}$ of the head of the pipe but level at the base. The material was selected so that self colour could be eliminated from the test. At the base of the pipe a clean bucket was placed to catch the outpouring from the pipe.

The colour blue was selected for the dye being tested (Figure 2). Other colours were rejected because they were close to the colours of the pipe, the collection bucket and the bucket used to mix up the dye.

Drain dye was mixed with tap water at ten times the concentration recommended by the manufacturers. A sample of the dyed water was retained for a colour match. Five litres of the dyed water was poured slowly into the sand at the head of the pipe so as to fill the $50 \mathrm{~mm}$ pipe above the sand. Over a 30-minute period the water level was topped up until all of the dyed water had passed into the sand.

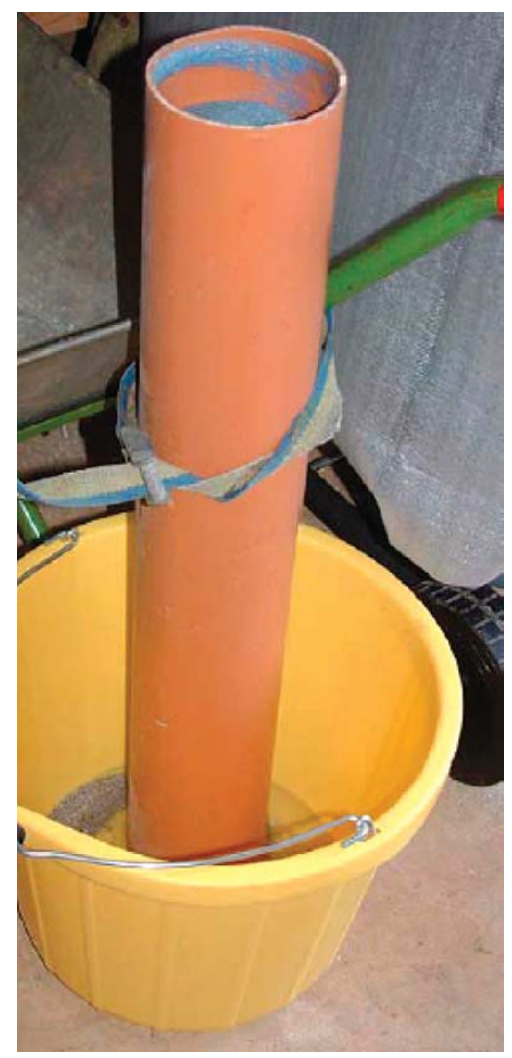

Figure 1: Vertical PVCu pipe filled with sand after testing. Water can be seen collected in the bucket after the test and the blue colour can be seen in the sand to the head of the pipe 


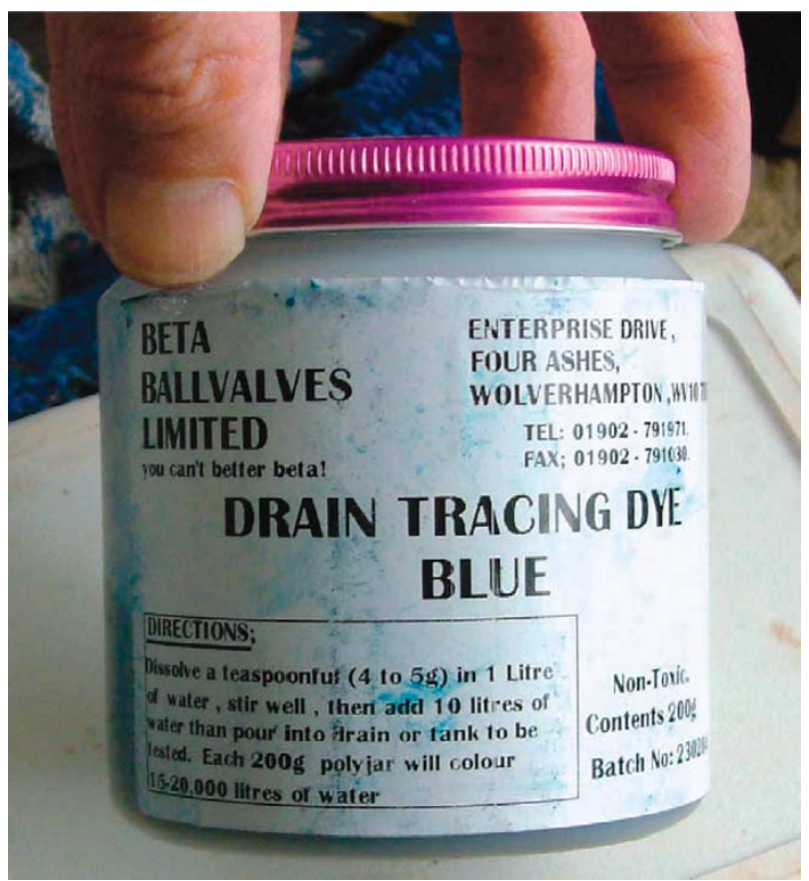

Figure 2: Drain dye used in the test

The pipe was left for two hours for the water to collect. The water that came out of the pipe was then matched for colour with the sample of the water that had been retained from the water poured into the pipe.

\section{RESULTS}

The water that came out of the pipe was without any trace of colour (Figure 3). The water that came through the test had a slightly cloudy trace, but no identifiable blue colorant as this had been retained in the sand (Figure 4).

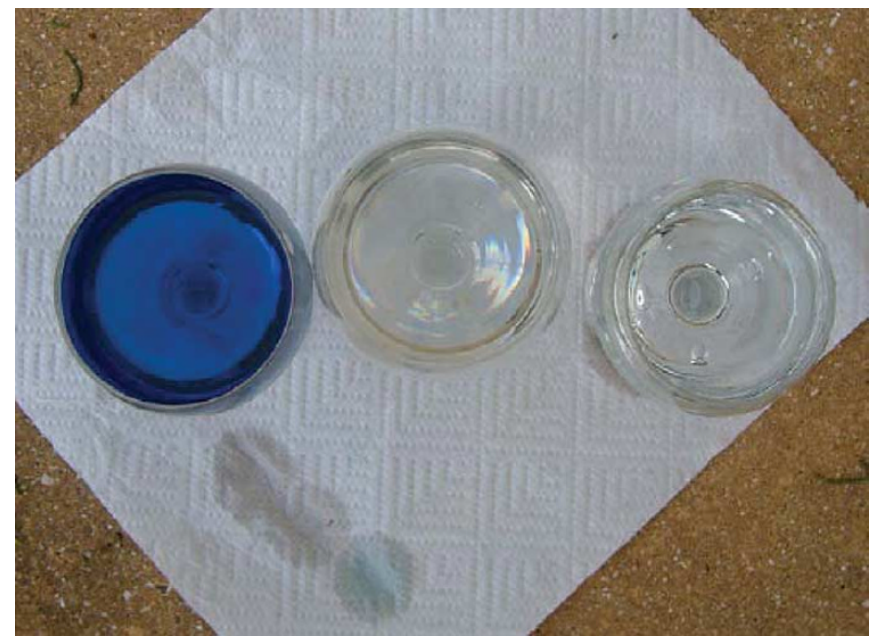

Figure 3: Left — dyed water prior to use; centre - water after passing through test; right - tap water used in test before being dyed 


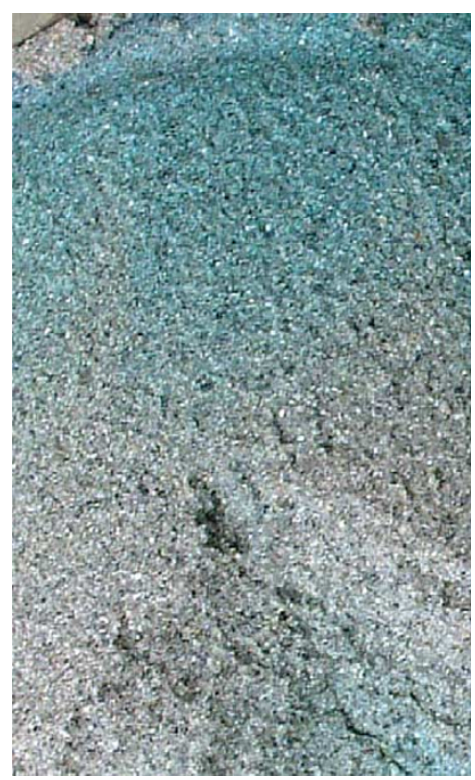

Figure 4: Example of colour retention in $300 \mathrm{~mm}$ of sand

This test is only for the effect of a sand-type ground. Representative samples of other ground materials are to be tested in the future.

\section{CONCLUSION}

In diagnosing drainage faults, drain tracing dye is only of use for identifying the linkage in continuous and connected pipe runs. It has no use as a diagnostic tool for tracing water flow if the dyed water flows through the ground. 